This is the post peer-review accepted manuscript of:

F. Guidi, N. Decarli, D. Dardari, F. Natali, E. Savioli and M. Bottazzi, (2016). A Low Complexity Scheme for Passive UWB-RFID: Proof of Concept. In IEEE Communications Letters, vol. 20, no. 4, pp. 676-679, April 2016.

https://doi.org/10.1109/LCOMM.2016.2530658

The published version is available online at: https://doi.org/10.1109/LCOMM.2016.2530658

(C) 2016 IEEE. Personal use of this material is permitted. Permission from IEEE must be obtained for all other uses, in any current or future media, including reprinting/republishing this material for advertising or promotional purposes, creating new collective works, for resale or redistribution to servers or lists, or reuse of any copyrighted component of this work in other works 


\title{
A Low Complexity Scheme for Passive UWB-RFID: Proof of Concept
}

\author{
Francesco Guidi Member, IEEE, Nicoló Decarli Member, IEEE, Davide Dardari Senior Member, IEEE, \\ Federico Natali, Enrico Savioli, Marco Bottazzi
}

\begin{abstract}
Passive UWB-RFID technology represents an emerging solution capable of guaranteeing extremely low energy consumption and high-accuracy localization at the same time. One of the most critical tasks is the acquisition of the tag code at reader side, which can be complex, time- and resourceconsuming when multiple UWB tags are deployed. This letter proposes a simple and effective approach, based on a specific assignment strategy of the tag code, which drastically simplifies code acquisition by guaranteeing high tag detection performance. A real system implementation adopting this strategy is shown to prove its feasibility in terms of real-time multiple tags detection and localization.
\end{abstract}

Index Terms-RFID, UWB, Backscatter, Tag Detection.

\section{INTRODUCTION}

Passive ultra-wideband (UWB)-radio-frequency identification (RFID) systems based on backscatter modulation are gaining interest for their capability of providing communication and precise localization with low energy consumption [1], [2]. Due to the absence of a transceiver in tags, there is no possibility to implement an anti-collision protocol for the multiple access. Consequently, a code division multiple access (CDMA) approach is usually considered, where a different spreading code is assigned to each tag [3]. Unfortunately, this approach requires code acquisition at reader side, which can be complex, time- and resources-consuming, becoming not feasible in many applications, especially if no additional synchronization procedures are considered [2]-[4]. In fact, the receiver must perform an extensive, and frequently impractical, code acquisition search with multiple de-spreaders to correctly detect the presence of asynchronous tags. Moreover, multi-tag interference is present and the related effects can completely affect the system operation due to near-far problems [3]. In this letter we propose an alternative solution that drastically simplifies the code acquisition procedure. In fact, with the presented scheme, fast (i.e., within one-symbol) detection of asynchronous tags is performed by preserving a lowcomplexity receiver thanks to a proper assignment strategy

This research was supported by the Italian Ministerial PRIN project GRETA (Grant 2010WHY5PR), the European H2020 project XCYCLE (Grant 635975), the H2020-EU.1.3.2 IF-EF Marie Curie action MAPS (Grant 659067).

F. Guidi is with CEA, LETI, MINATEC Campus, 38054 Grenoble, France (e-mail: francesco.guidi@cea.fr).

N. Decarli and D. Dardari are with the DEI, University of Bologna, Italy (e-mail: \{nicolo.decarli, davide.dardari\}@unibo.it).

F. Natali, E. Savioli and M. Bottazzi are with Datalogic IP Tech S.r.l., Bologna, Italy (e-mail: ffederico.natali, enrico.savioli, marco.bottazzi\}@datalogic.com).

Digital Object Identifier 10.1109/LCOMM.2016.2530658 of the tag code. Despite this solution is suitable only with few tags simultaneously present in the monitored environment, there are several applications where the high-accuracy localization is mandatory and this limited number is not an issue. An example is represented by sorting systems for items moving on conveyor-belts, e.g., luggage in airports, where a few tags are simultaneously present in the conveyor portion where the localization is performed. Even in completely asynchronous conditions, this solution is able to guarantee an interference-free detection without a significant performance loss. Experimental results obtained from an ad-hoc realization of the system show the feasibility of multi-tag detection.

The main contributions of this letter are: (i) the introduction of a new low-complexity solution for multi-tag deployment of UWB-RFID systems which avoids the use of a dedicated synchronization channel and an extensive code acquisition; (ii) the presentation of the first practical test-bed showing the realtime detection and ranging of moving UWB-RFID tags in a real application context. The rest of the letter is organized as follows. In Sec. II the UWB-RFID system architecture is revisited; in Sec. III-A the proposed tag code assignment strategy is presented; finally, Sec. IV shows the experimental results obtained in a multi-tag scenario.

\section{System ARChitecture}

In the following, the UWB backscatter mechanism and the receiver architecture, exploited in Sec. III-A, are re-visited.

\section{A. UWB Backscatter Communication}

The considered architecture foresees the deployment of a reader that interrogates the surrounding passive tags in order to identify them through the analysis of the backscattered signal. Without loss of generality, we account for the transmission of a continuous sequence of pulses, i.e.,

$$
s(t)=\sum_{l=-\infty}^{\infty} p\left(t-l T_{\mathrm{p}}\right)
$$

where $p(t)$ is the transmitted UWB pulse repeated with period $T_{\mathrm{p}}$. When a reader transmits the interrogation signal (1), each tag sends its information back to the reader by modulating the backscatter signal through a proper variation of the load connected to its antenna using a simple UWB switch (backscatter modulation) [1]. The useful signal part is called antenna mode scattering and it is buried by the clutter (i.e., the response of the environment) and the structural mode 
scattering, which contains all reflections due to the antenna itself [5]. To mitigate clutter and interference, an antipodal balanced code $c_{n}^{(k)} \in\{-1,1\}$, for $n=0,1, \ldots, N_{\mathrm{c}}^{(k)}-1$, is assigned to the $k$ th tag. Such code drives the switch that changes the antenna load (short or open circuit) every $T_{\mathrm{c}}^{(k)}$ seconds, where $T_{\mathrm{c}}^{(k)}=N_{\mathrm{pc}}^{(k)} T_{\mathrm{p}}$ is the $k$ th tag chip time with $N_{\mathrm{pc}}^{(k)}$ being the number of pulses per chip. For further convenience, we define the new sequence $\tilde{c}_{l}^{(k)} \triangleq c_{\left\lfloor l / N_{\mathrm{pc}}^{(k)}\right\rfloor}^{(k)}$ for $l=0,1, \ldots, N_{\mathrm{s}}-1$, with $\tilde{c}_{l+N_{\mathrm{s}}}^{(k)}=\tilde{c}_{l}^{(k)}$, and where $\lfloor\cdot\rfloor$ denotes the floor operation. Thus, we define $N_{\mathrm{s}}=N_{\mathrm{c}}^{(k)} N_{\mathrm{pc}}^{(k)}$ as the number of pulses per symbol, and it is the same for all tags.

Note that, for each transmitted pulse, the signal backscattered by the tag propagates back to the reader's antenna through the reader-tag link. In this way, the tag component results to be spread by the code $\left\{\tilde{c}_{n}^{(k)}\right\}$, thus enabling the detection of tag signal and the clutter cancellation, as described in the following section.

\section{B. Receiver Architecture}

In order to allow the discrimination of the useful tag signal from the other contributions, a de-spreading procedure at the reader side is operated. According to the processing scheme described in [3], the receiver performs the coherent accumulation of the received signals corresponding to the $N_{\mathrm{s}}$ transmitted pulses $p(t)$ within a symbol. Note that tags' code generators at reader side used for de-spreading are completely asynchronous with respect to that of tags. Thus, there is an uncertainty on the offset (phase) of the tag's spreading code with respect to the local generators of the reader. A possible solution is to perform the detection jointly with code acquisition by employing parallel de-spreaders, each tuned to a differently shifted version of the sequence $\left\{\tilde{c}_{l}^{(\hat{k})}\right\}$, where $\hat{k}$ denotes the tag the receiver aims to detect [3]. In this way, a dedicated synchronization channel is avoided. For the proposed scheme, we consider code acquisition with $N_{\text {span }}$ shifts (i.e., $N_{\text {span }}$ parallel de-spreaders) and step $\Delta$, which determines an overall code acquisition window $\mathrm{AW}=\Delta N_{\text {span }}$. Note that $N_{\text {span }}$ is upper limited by the sustainable receiver complexity. For this reason, as will be detailed in the following, codes robust to asynchronous offsets have to be adopted in order to maintain the system complexity affordable.

Without loss of generality, we consider the detection of the $\hat{k}$ tag (useful tag) by observing the first $N_{\text {s }}$ pulses, i.e., a symbol. Denote $r_{\text {reader }}(t)=w(t)+n(t)$ the received signal, where $w(t)$ contains the response of all tags and of the environment to the interrogation signal, and $n(t)$ is a zero-mean Gaussian random process. According to [3], first the received signal is filtered to eliminate the out-of-band noise, obtaining $\tilde{r}(t)=$ $\tilde{w}(t)+\tilde{n}(t)$. Once the signal is filtered, it is de-spread giving $N_{\text {span }}$ outputs corresponding to $N_{\text {span }}$ different code shifts, obtaining

$$
y_{n}(t)=\sum_{l=0}^{N_{\mathrm{s}}-1} \tilde{c}_{l+n \Delta}^{(\hat{k})} \tilde{r}\left(t+l T_{\mathrm{p}}\right)
$$

with $t \in\left[0, T_{\mathrm{p}}\right]$ and $n=1,2, \ldots, N_{\text {span. In }}$. Ine absence of parallel code acquisition (i.e., only 1 de-spreader) it is
$N_{\text {span }}=1$ and $\Delta=0$. It is possible to decompose (2) as $y_{n}(t)=x_{n}(t)+z_{n}(t)$, with $z_{n}(t)$ indicating the noise term. The detection in parallel of $N_{\text {tag }}$ tags requires replicating for each tag the same receiver structure. Since the clutter component is canceled through the de-spreading if the tag code $\left\{c_{l}^{(\hat{k})}\right\}$ is exactly balanced (i.e., with the same number of \pm 1 ) [3], we adopt balanced codes, for which $x_{n}(t)$ reduces to

$$
x_{n}(t)=\sum_{l=0}^{N_{\mathrm{s}}-1} \tilde{c}_{l+n \Delta}^{(\hat{k})} \sum_{k=1}^{N_{\text {tag }}} \omega^{(k)}\left(t+l T_{\mathrm{p}}\right) \quad t \in\left[0, T_{\mathrm{p}}\right]
$$

where the filtered single-tag channel response to the interrogation signal is $\omega^{(k)}(t)$. Referring to the partially non-coherent receiver scheme described in [3], energy bins are obtained for each de-spreader output $y_{n}(t)$ and over a period $T_{\mathrm{p}}$ as follows

$$
e_{n, b}=\int_{(b-1) T_{\mathrm{ED}}}^{b T_{\mathrm{ED}}}\left[y_{n}(t)\right]^{2} d t
$$

with $n=1,2, \ldots, N_{\text {span }}, b=1,2, \ldots, N_{\text {bin }} . T_{\mathrm{ED}}$ denotes the integration time, and $N_{\text {bin }}=\left\lfloor T_{\mathrm{p}} / T_{\mathrm{ED}}\right\rfloor$ is the number of bins at the output of the energy detector (ED). Therefore, a $N_{\text {bin }} \times N_{\text {span }}$ energy matrix $\mathbf{E}=\left\{e_{n, b}\right\}$ is given by all the ED bins. Each element $e_{n, b}$ of the energy matrix is then compared with a threshold, and if this is exceeded by at least one element the tag is considered detected. Note that the bin index gives information about the time-of-arrival (TOA) of the tag signal, thus enabling accurate reader-tag ranging. The choice of $N_{\text {span }}, \Delta$ and code assignment strategy allows to reach the best trade-off between receiver complexity and performance, as investigated in the next section.

\section{Tag Codes Assignment Strategy}

We now propose a simple and effective scheme, based on a specific tag code assignment, which leads to obtain fast tag detection while preserving a low system complexity.

\section{A. Codes Assignment}

Consider the system architecture of Sec. II, with one reader and fully asynchronous tags. The idea for a low-complexity multi-tag detection, without experiencing detrimental interference effects, is based on the adoption of codewords having the alternation of the chip +1 and -1 at different rates, i.e., with a different number of pulses per chip $N_{\mathrm{pc}}^{(k)}$ for each tag. According to this scheme, considering for example $N_{\mathrm{pc}}^{(k)}=1$ and $N_{\mathrm{pc}}^{\left(k^{\prime}\right)}=4$, the codes result constructed as:

$$
\begin{aligned}
& \tilde{c}^{(k)}=c^{(k)}=\{\underbrace{+1,-1}_{1 \text { pulse per chip }},+1,-1,+1,-1,+1,-1,+1, \ldots\} \\
& \tilde{c}^{\left(k^{\prime}\right)}=\{\underbrace{+1,+1,+1,+1,-1,-1,-1,-1}_{4 \text { pulses per chip }},+1,+1,+1,+1, \ldots\} .
\end{aligned}
$$

These codes are cyclic orthogonal Walsh Hadamard sequences, which are a subset of Walsh-Hadamard codes, and show a periodic behavior, where the period corresponds to twice the chip time. The adoption of these cyclic codes leads to reduce the code acquisition window (i.e., the receiver 
complexity) which can now be limited within a chip. Then, at reader side, for the detection of each tag a specific couple $\left(N_{\mathrm{span}}^{(k)}, \Delta^{(k)}\right)$ is assigned, implying the adoption of different de-spreaders steps $\Delta^{(k)}$ according to the specific tag to be detected. We adopt $N_{\mathrm{span}}^{(k)} \leq N_{\mathrm{pc}}^{(k)}$ and $:^{1}$

$$
\begin{aligned}
& \mathrm{AW}^{(k)}=N_{\mathrm{pc}}^{(k)}, \quad \text { for } \quad N_{\mathrm{span}}^{(k)}>1 \\
& \mathrm{AW}^{(k)}=0, \quad \text { for } \quad N_{\mathrm{span}}^{(k)}=1 \text {. }
\end{aligned}
$$

Then, the step $\Delta^{(k)}$, defined only for $N_{\text {span }}>1$, is given by the relation $\Delta^{(k)}=N_{\mathrm{pc}}^{(k)} / N_{\mathrm{span}}^{(k)}$. Note that for the considered case with a symbol length $N_{\mathrm{s}}$ and a minimum number of pulses per chip $N_{\mathrm{pc}}^{(\min )}=\min _{k}\left\{N_{\mathrm{pc}}^{(k)}\right\}$, the maximum number of different codewords (i.e., the maximum number of tags simultaneously active) is given by $N_{\max }=\log _{2}\left(N_{\mathrm{s}} / N_{\mathrm{pc}}^{(\min )}\right)$. As an example, this means that 13 tags can be managed at the same time with $N_{\mathrm{s}}=8192$ and $N_{\mathrm{pc}}^{(\min )}=1$.

\section{B. Codes Performance}

One important requirement for reliable detection and interference mitigation is the availability of a high processing gain for all possible codes. ${ }^{2}$ Thus, we tested the proposed sequences considering tags with $N_{\mathrm{s}}=8192$ and different codewords/parameters, i.e., $N_{\mathrm{pc}}^{(k)}, \Delta^{(k)}$ and $N_{\text {span }}^{(k)}$ for each tag. In Table I, results are reported considering the codes' autocorrelation function, determining the effective process gain, defined as

$$
\mathcal{A}_{n, m}=\frac{1}{N_{\mathrm{s}}} \sum_{l=1}^{N_{\mathrm{s}}} \tilde{c}_{l+n \Delta(k)}^{(k)} \cdot \tilde{c}_{l-m}^{(k)}
$$

where $m=1,2, \ldots, N_{\mathrm{s}}$ is the set of possible code shifts due to the asynchronous conditions. Specifically, the average and the maximum processing gain losses, computed over all possible code shifts, are defined as

$$
\begin{aligned}
& \mathrm{PG}_{\text {loss }}^{(\text {avg })}=\frac{1}{N_{\mathrm{s}}} \sum_{m=1}^{N_{\mathrm{s}}}\left(\max _{n} \mathcal{A}_{n, m}\right)^{-1} \\
& \mathrm{PG}_{\text {loss }}^{(\max )}=\max _{m}\left(\max _{n} \mathcal{A}_{n, m}\right)^{-1}=\frac{N_{\mathrm{pc}}}{N_{\mathrm{pc}}-2\left\lfloor\frac{\Delta^{(k)}}{2}\right\rfloor} .
\end{aligned}
$$

From Table I, the presence of a loss in the processing gain is evident for tags adopting higher $N_{\mathrm{pc}}$ with $N_{\mathrm{span}}=8$. In fact, the lower the number of de-spreaders, the coarser the search of the code offset. The processing gain loss is in general low (about $0.57 \mathrm{~dB}$ and $0.28 \mathrm{~dB}$ on average for the considered cases). Moreover, if we want to achieve a higher processing gain also for the detection of the disadvantaged tags (i.e., tags with high $N_{\mathrm{pc}}^{(k)}$ ), it is possible to increase the number of de-spreaders for that tags, without the need of enlarging the acquisition window of all tags. The main advantage of the considered codes is their orthogonality, which translates into the complete absence of multi-tag interference, also in completely asynchronous scenarios. In fact, the cross-correlation

\footnotetext{
${ }^{1}$ Without loss of generality, it is here assumed $N_{\mathrm{span}}^{(k)}, \Delta^{(k)}$ and $N_{\mathrm{pc}}^{(k)}$ being power of 2 (e.g., $N_{\mathrm{pc}}^{(1)}=8, N_{\mathrm{pc}}^{(2)}=16, N_{\mathrm{pc}}^{(3)}=32, \ldots$ ).

${ }^{2}$ Note that in a perfectly synchronous system the accumulation of the received signal portions according to (2) gives a process gain $N_{\mathrm{S}}$.
}

TABLE I

Processing GAIN LOSS FOR $N_{\mathrm{s}}=8192$

\begin{tabular}{c|cccccc}
\multicolumn{7}{c}{ Tags Parameters and Processing Gain } \\
\hline$N_{\mathrm{pc}}^{(k)}$ & 1 & 8 & 512 & 512 & 4096 & 4096 \\
$N_{\mathrm{span}}^{(k)}, \Delta^{(k)}$ & $(1,0)$ & $(8,1)$ & $(8,64)$ & $(16,32)$ & $(8,512)$ & $(16,256)$ \\
$\mathrm{PG}_{\text {loss }}^{(\text {avg })}[\mathrm{dB}]$ & 0 & 0 & 0.57 & 0.28 & 0.57 & 0.28 \\
$\mathrm{PG}_{\text {loss }}^{(\text {max })}\left[{ }^{\mathrm{dB}}\right]$ & 0 & 0 & 1.16 & 0.56 & 1.16 & 0.56
\end{tabular}

function between two codewords is $\boldsymbol{\theta}^{\left(k, k^{\prime}\right)}=\left\{\theta_{n, m}^{\left(k, k^{\prime}\right)}\right\}=\mathbf{0}$, $\forall k \neq k^{\prime}, \forall m=1,2, \ldots, N_{\mathrm{s}}$ and $\forall n=1, \ldots, N_{\text {span }}$, with

$$
\theta_{n, m}^{\left(k, k^{\prime}\right)}=\sum_{l=1}^{N_{\mathrm{s}}} \tilde{c}_{l+n \Delta^{(k)}}^{(k)} \cdot \tilde{c}_{l-m}^{\left(k^{\prime}\right)} .
$$

In the following, an experimental validation of the lowcomplexity multi-tag RFID scheme based on the proposed codes is shown for a real application.

\section{EXPERIMENTAL VALIDATION}

We now describe the measurement scenario and the obtained experimental results, in order to validate the aforementioned UWB RFID scheme.

We consider the reader-tag architecture described in [6]. The realized reader has to cope with the high-complexity of the signal processing algorithms and with the high-level of parallelism required for tag detection, identification, and localization. All these tasks are accomplished by an ad-hoc realized TX/RX RF front-end and an FPGA, which performs the digital base-band signal processing by guaranteeing: (i) the coherent integration of the backscattered pulses considering the tag codes according to (2) (de-spreading); (ii) the energy profile evaluation according to (4) and the computation of the round-trip time of the received signal.

The FPGA controls also the emission of UWB root-raised cosine pulses from the TX board, centered at $4.5 \mathrm{GHz}$ with $700 \mathrm{MHz}$ bandwidth. The RX board samples the in-phase and in-quadrature received signals and forwards them to the FPGA, where signal processing is performed in order to construct the energy matrix $\mathbf{E}$ of eq. (4) and to provide a TOA estimate to the microprocessor. The receiver structure described in Sec. II is replicated for the $N_{\text {tag }}$ tags to be detected in parallel. The microprocessor packages the data which are successively sent to the central unit via the Ethernet connection. Tags were realized by connecting a UWB antenna with a switch changing its status periodically according to the codes described in Sec. III-A. The reader was placed over a conveyor belt, in order to emulate a real system devoted to the sorting of goods in industrial/logistic scenarios (Fig. 1). Two antennas, one transmitting and one receiving placed in quasi-monostatic configuration, were pointed towards the direction of arrival of tags attached over paper boxes. In this way, the estimation of the reader-tag distance allowed determining the tag position over the conveyor and then the tags order of arrival. 


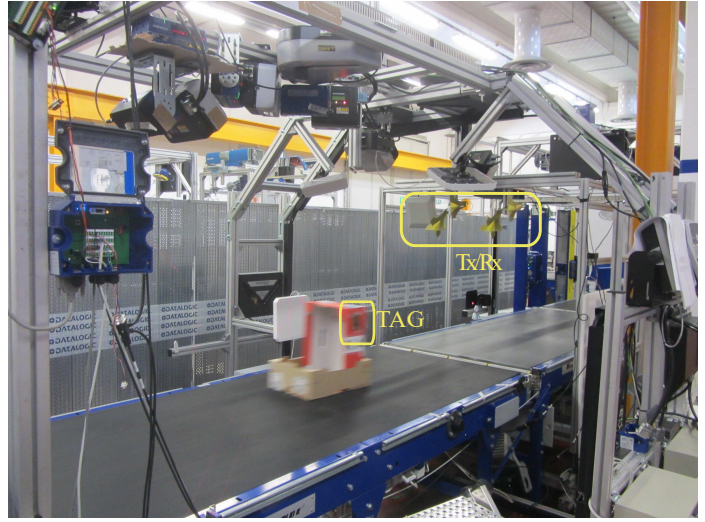

Fig. 1. Conveyor-belt scenario adopted for measurements.

\section{A. Results}

Two kinds of tests were performed, with static and moving conveyor, in order to test both the ranging capabilities and the real-time multi-tags detection performance.

1) Ranging Performance: With the conveyor still, we accounted for one tag (here named as \#1) placed at different distances, from $1.5 \mathrm{~m}$ to $4.8 \mathrm{~m}$ spaced apart by $0.15 \mathrm{~m}$, and repeating the measure 500 times per position.

Results in Fig. 2 confirmed that the maximum reader-tag reading distance is up to $4.80 \mathrm{~m}$. For each position, the maximum error committed was in the order of the bin resolution, corresponding to $15 \mathrm{~cm}$ in backscattering due to the chosen $T_{\mathrm{ED}}=1 \mathrm{~ns}$. At greater distances the backscattered signal energy was low (see Fig. 2), and it was not possible to define a threshold for providing a false alarm probability lower than $10^{-2}$ as well as a desired detection rate of at least $90 \%$.

2) Tests with Moving Conveyor: We then performed tests by placing 4 tags at different distances, with spacing comprised between 30 and $70 \mathrm{~cm}$, on a conveyor moving at $1 \mathrm{~m} / \mathrm{s}$. We assigned codes with $N_{\mathrm{pc}}^{(1)}=1, N_{\mathrm{pc}}^{(2)}=8, N_{\mathrm{pc}}^{(3)}=512$, and $N_{\mathrm{pc}}^{(4)}=4096$ to the tags, by fixing $N_{\mathrm{span}}^{(1)}=1$ and $N_{\text {span }}^{(2)}=N_{\text {span }}^{(3)}=N_{\text {span }}^{(4)}=8$. As previously shown, in order to keep the system complexity affordable, we fixed $N_{\text {span }}^{(k)}$ and thus the number of correlators. Note that, in the presented results, according to the design proposed in [3], a bin-dependent threshold (green line) has been adopted instead of a bin-independent threshold (red line), in order to obtain a fixed detection rate and to preserve an overall false alarm rate of $10^{-2}$. Such a threshold accounts not only for the receiver noise, but also for the expected tags energy related to a certain bin index (i.e., reader-tag distance) and to the system nonidealities. In fact, in our scenario, the dependence on the bin has been determined by considering the energy received by tag \#1 placed at different distances from the reader antennas.

In Fig. 2 an example of real-time detection with moving conveyor is reported, where tags are correctly sorted. Specifically, for each tag the peak of the energy matrix $\mathbf{E}$ measured by the FPGA is shown in correspondence of its bin index.

Final tests were performed for 500 times (i.e., for each time, the tag detection was performed exploiting a circular track). A false alarm rate below $10^{-2}$ and a detection rate of $100 \%$

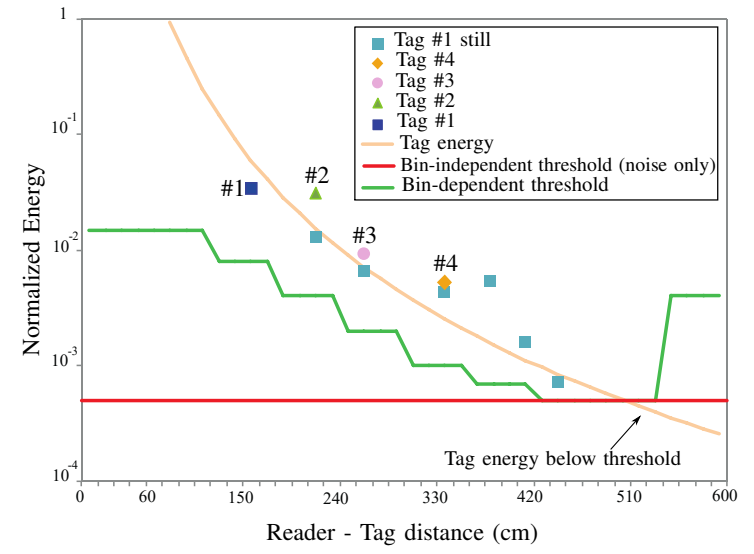

Fig. 2. Example of tags sorting with moving and static conveyor.

over 500 acquisitions were obtained for a moving conveyor, by imposing a maximum reading distance of $2 \mathrm{~m}$ in order to work in the highest signal-to-noise ratio (SNR) region and improve the system robustness with respect to receiver non-idealities. For each test, tags were correctly sorted. Such results, obtained in a real practical scenario, confirmed the possibility to adopt this simple and effective solution for multi-tag detection and sorting/localization by guaranteeing reliable performance. In addition, it represents the world first experimental proof of concept of passive UWB RFID technology.

\section{CONCLUSION}

This letter showed a new low-complexity solution for fast detection of multiple passive UWB-RFID tags avoiding the need of a dedicated time- and resource-consuming code acquisition and the implementation of complex receiver structures, thanks to the adoption of cyclic orthogonal codes. Experimental results collected on a real moving conveyor belt highlighted for the first time the feasibility of such a scheme, which represents a novel and viable solution for several applications where high-accuracy identification and localization are required.

\section{ACKNOWLEDGMENT}

The authors would like to thank the SELECT project partners for the fruitful cooperation and the hardware realization.

\section{REFERENCES}

[1] D. Dardari et al., "Ultrawide bandwidth RFID: The next generation?" Proc. IEEE, vol. 98, no. 9, pp. 1570-1582, Sep. 2010.

[2] A. Ramos, A. Lazaro, and D. Girbau, "Semi-passive time-domain UWB RFID system," IEEE Trans. Microw. Theory Tech., vol. 61, no. 4, pp. 1700-1708, Apr. 2013.

[3] F. Guidi et al., "Detection of multiple tags based on impulsive backscattered signals," IEEE Trans. Commun., vol. 62, no. 11, pp. 3918-3930, Nov. 2014.

[4] D. Arnitz, U. Muehlmann, and K. Witrisal, "UWB ranging in passive UHF RFID: proof of concept," Electronics Lett., vol. 46, no. 20, pp. 1401-1402, Sep. 2010.

[5] S. Hu et al., "Backscattering cross section of ultrawideband antennas," IEEE Antennas Wireless Propag. Lett., vol. 6, pp. 70-73, Mar. 2007.

[6] E. Savioli et al., "Semi-passive UHF-UWB RFID: Architecture and localization performance," in Proc. IEEE Int. Conf. Commun. Workshops, Budapest, Hungary, Jun. 2013, pp. 1-5. 